\title{
HECATEO Y LAS INSCRIPCIONES GRIEGAS MÁS ANTIGUAS DE LA PENÍNSULA IBÉRICA
}

\author{
POR
}

\author{
ELVIRA GANGUTIA ELÍCEGUI \\ Instituto de Filología, CSIC, Madrid
}

\section{RESUMEN}

En este trabajo comparamos varios fragmentos de Hecateo de Mileto con algunas de las más antiguas inscripciones griegas halladas en España, lo que, creemos, contribuirá a arrojar alguna nueva luz sobre dichos textos.

\section{SUMMARY}

We have tried in this paper to make a comparison between some of the fragments of Hecateus of Milet and some of the most ancient Greek inscriptions found in Spain, with the idea that it may contribute to the elucidation of the aforesaid texts.

1. El descubrimiento en suelo hispano y publicación en los últimos años de cierto número de inscripciones griegas notablemente arcaicas ha generado considerable y lógica expectación. El interés por estos epígrafes en variados soportes, entre los que hay que resaltar los plomos, incluido el llamado de Pech-Maho, proviene no sólo desde el punto de vista de la disciplina epigráfica en cuanto tal, sino por lo que tienen de posibilidad de contraste con otras fuentes. En este trabajo nos ceñiremos a los más antiguos epígrafes, especialmente aquellos que en algún momento han sido fechados entre el vi/v a.C. ${ }^{1}$, tratando de elucidar en qué medida estos textos in-

1 En principio los recogidos en $I G A I=$ Inscriptiones graecae antiquissimae Iberiae, ed., trad. y com. de Rodríguez Somolinos, H., 333 ss. en Gangutia, E., La Península Ibérica en los autores griegos de Homero a Platón. Testimonia Hispaniae Antiqua II A. Eds. J. Mangas y D. Plácido, Madrid, 1998, XVI + 332 e índices (en adelante sólo THA y página); éstas y otras inscripciones en un marco cronológico más amplio en $E G H=$ de Hoz, M.P., «Epigrafía griega de Hispania», Epigraphica, 59, 1997, 29-96. Tanto en IGAI como en $E G H$ se documenta cada inscripción con las diferentes ediciones, dataciones propuestas y abundante, clasificada y crítica bibliografía. Sobre estas ediciones, básicamente sobre $I G A I$ y su traducción, con mínimos retoques, está hecha la selección de textos utilizados en este trabajo que ofrecemos como APÉNDICE DE TEXTOS EPIGRÁFICOS UTILIZADOS al final del presente trabajo.

Para las citas de autores y obras antiguas se siguen las ediciones publicadas en AA.VV., Diccionario Griego Español, Madrid, CSIC, 1991, III, páginas XXIII ss., puestas al día en http:/ www.filol.csic. es/depts/dge/lst/lst-int.htm, ciden en el conocimiento de uno de los «padres» de la historia y de la geografía, Hecateo de Mileto, cuyos escuetos y debatidos fragmentos contienen desde época arcaica notables datos sobre la península ibérica, las Baleares y un mundo transpirenaico que alcanza hasta Etruria. A su vez, a partir de ellos y otros del mismo autor referidos a zonas más alejadas, podremos también conseguir una perspectiva mejor para la interpretación de las mencionadas inscripciones, ya que puede decirse que grosso modo gran número de ellas coinciden con la datación del historiador, por otro lado todavía no precisada con absoluta exactitud ${ }^{2}$.

Se ha creído ver en alguna de las más antiguas inscripciones griegas de la Península explicaciones

mientras no se indique lo contrario. Agradezco a H. Rodríguez Somolinos el préstamo de artículos inéditos, así como el acceso a la amable carta del Prof. A.M. de Faria; a Eugenio Luján por la lectura del original y sus autorizadas observaciones, así como a J.A. Berenguer; a J. Rodríguez Somolinos, por su gran ayuda en la edición informática.

${ }^{2}$ Se tiende a situar el acmé de Hecateo en los años 20 del VI a.C; tal vez algo más tarde escribiría las Genealogías y Periégesis, obras en cualquier caso complementarias y en las que en época posterior probablemente se han ido introduciendo subdivisiones: ver $R E$ s.v. Hekataios (Jacoby), editor que seguimos para Hecateo en Die Fragmente der griechischen Historiker. Leiden, $1957^{2}, \mathrm{I}, \mathrm{n}^{\circ} 1$, citado sólo por la numeración de los Fr.; su comentario (citado Jacoby comm. y página) de la misma fecha y lugar, I, 318 ss. sigue siendo útil. Cf. también Hecatei Milesii fragmenta (intr. ed., trad. G. Nenci), Florencia, 1954 (citado por $n^{\circ}$ de Fr. ... Nenci), con bibliografía; para estudios generales y a veces con especial mención de la Península, ver Pearson, L., Early Ionian Historians, Oxford, 1939, 25-108; los diferentes trabajos de Tozzi, P., «Studi su Ecateo di Mileto», entre los que destacamos el II «Ecateo e la cultura ionica», Athenaeum, 41, 1963, 318-

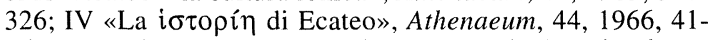
76; von Fritz, K., Die griechische Geschichtsschreibung. Berlín, 1967, I, 48-76; II, 32-53; Lasserre, F., «L'historiographie grecque à l'époque archaïque», Quaderni di storia, 1976, 113-142; Lens, J., en Historia de la literatura griega. Ed. J.A. López Férez, Madrid, 1988, 263-265; Lanzillotta, E., «Geografia e storia da Ecateo a Tucidide», CISA, 1988, 29; de Hoz, J., «Las fuentes escritas sobre Tartessos», Tartessos. Arqueología protohistórica del Bajo Guadalquivir. Ed. M.E. Aubet, Sabadell, 1989, 28 ss; West, St., «Herodotus' portrait of Hecataeus», JHS, 111, 1991, 144-160; Olmos Romera, R., «Las huellas griegas en el ámbito peninsular: los últimos hallazgos, historiografía y método», Griegos en $O c$ cidente. Ed. F. Chaves Tristán, Sevilla, 1992, 157; Nicolai, R., «Pater semper incertus. Appunti su Hecateo», QUCC, 56, 1997, 143-164. Ver nuestro THA 136-155. 
muy concretas de los Fragmentos de Hecateo ${ }^{3}$. Esto puede ser excesivo, y pensamos más bien que, aunque hay en estas inscripciones muchos datos que consolidan o abren perspectivas sobre la visión del mundo mediterráneo en expansión tal como se manifiesta en general en Hecateo y otras fuentes literarias de la época, lo que en aquellas encontramos son ciertas claves y complementariedad.

Los fragmentos de la Periégesis de Hecateo que contienen nombres concretos de la Península, aunque alguna vez describan el interior o pasen a islas, como las Baleares, tienen como hilo conductor una a modo de línea de puntos costera que va desde el Océano (Atlántico), sobrepasando las Columnas de Hércules en dirección este y nordeste hasta atravesar los Pirineos y que, dando la vuelta al Mediterráneo, vuelve otra vez al Océano por las costas norteafricanas ${ }^{4}$. Este rumbo en el sentido de las «manecillas del reloj» se desprende, en unos casos, por posición relativa a las Columnas de Hércules; en otros porque se han mantenido segmentos concretos del texto hecateico con la secuencia $\mu \varepsilon \tau \grave{\alpha} \quad \delta \grave{\varepsilon} \quad \ldots$ $\mu \varepsilon \tau \grave{\alpha} \delta \grave{\varepsilon}$; y en otros, gracias a testimonios de autores posteriores en los que se conservan huellas de Hecateo, como en Herodoro de Heraclea o Avieno ${ }^{5}$.

Las inscripciones griegas más antiguas halladas en la Península no contradicen este trazado. Además, las alusiones encontradas en ellas rebasan la localización concreta de su hallazgo y amplían considerablemente el área geográfica, de manera que encuentran su eco en la descripción hecateica en forma más general e incluso permiten avanzar en el conocimiento del modo de componer la obra de nuestro autor.

2. Los epígrafes encontrados en Andalucía se concentran en dos zonas concretas: en Huelva, IGAI $10,13(E G H 22.1,2)$ [de mediados del vi y finales del vir a.C. respectivamente] e IGAI 9 [vi a.C.]; y en Málaga IGAI 12, 14 (EGH 16.1, 17.1) [VII a.C. y 630-550 a.C. respectivamente] e IGAI 11 [vi a.C.]. Como puede verse, para algunas de ellas se han pro-

\footnotetext{
${ }^{3}$ Musso, O., «Il piombo inscrito di Ampurias: note lin guistiche e datazione», Empùries, 48-50 (2), 1986-89 [1993], 156-59: lee en IGAI 1.7, (EGH 2.14.7) $\beta \grave{\alpha} \varsigma \pi \varepsilon \delta[o v]$ 'E $\lambda_{1}[\beta v \rho \gamma i \omega v$ (cf. Hecateo Fr. 38) y ve en el texto alusiones al azufre y el estaño, así como a los carpetanos. Críticas en $I G A I 1$ y en $E G H 2.14$.

${ }^{4}$ Hasta @írm o Tánger, Hecateo Fr. 354.

5 En el ámbito de la Península, Hecateo $F r .43$, 48; fuera de ella, ver $F r .265,266$, cf. el mismo método en Herodoro $2 \mathrm{a}$, (ver nuestro THA pp. 149, 276, nn. 299, 305). Hecateo es el primer autor griego reconocido como fuente por Avieno, Ora 42. Cf. Aly, W., «Die Entdeckung des Westens», Hermes, 62, 1927, [pp. 299-341], pp. 398 ss.; se ha querido ver en el punteado de nombres citados por Hecateo una sombra de rutas foceas: Olmos Romera, R., cit. (n. 2), 157.
}

puesto fechas anteriores a Hecateo e incluso a la famosa visita de los foceos a Argantonio, producida cuando empieza «el auge del Medo» según Heródoto en 1.163: nos aproximarían a una fase próxima al «viaje de Coleo» descrito en Heródoto 4.152, remontando incluso a una época «eriteica» de la que tendríamos rastros en Hesíodo Th. 290 y Estesícoro 184 p.154 Davies, tradición que Hecateo tuvo en cuenta, aunque con una interpretación particular, como puede verse por su $F r .26$.

Las dos concentraciones epigráficas documentadas para Andalucía encuentran cierto eco en Hecateo. La situación allende Gibraltar correspondería grosso modo a las noticias que transmite sobre la ciudad de 'E $\mathrm{\lambda} \imath \beta u ́ \rho \gamma \eta$ Elibirge o Elibirga $(F r .38)$ e "I $\beta \cup \lambda \lambda \alpha$ Ibila (Fr. 45 Nenci), ambas en ámbito tar-

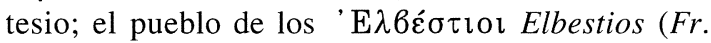
40) y la ciudad de $\mathrm{K} \alpha \lambda \alpha \dot{\theta} \eta$ Calate o Calata ( $F r$. 39). Sobrepasado Gibraltar hacia el Mediterráneo, Hecateo menciona el pueblo de los M $\alpha \sigma \tau \imath \eta v o i ́$ Mastienos o Mastianos ( $F r$. 40), cuyas ciudades en el entorno de Málaga son reseñadas por el logógrafo con cierta minuciosidad ( $\Sigma u ́ \alpha \lambda ı \varsigma$ Sualis Fr. 25 Nen-

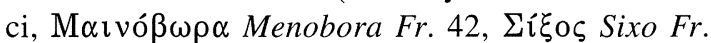

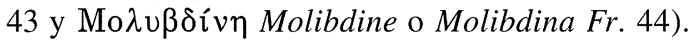

Gracias a las preciadas inscripciones es posible conectar algunos datos. Recientemente ${ }^{6}$ habíamos propuesto que la terminación $-\theta \eta$ de la $K \alpha \lambda \dot{\alpha} \theta \eta$ transmitida por Hecateo, podía ser un formante femenino con cierta correspondencia en $\Sigma \alpha \imath \gamma \alpha \dot{\alpha} \nu \theta \eta \imath$ Saigante o Seganta del plomo de Ampurias IGAI 1 ( $E G H$ 2.14). Queremos añadir ahora que la forma masculina de dicha terminación podría encontrarse en la inscripción de un vaso samio o foceo, ].v vın$\theta \omega \imath[$, «Fulano este vaso don]ó a Nieto[» de IGAI 10 (EGH 22.1) procedente de Huelva. Contiene sin duda un antropónimo no griego, tal vez procedente de un lugar no muy alejado de la $\mathrm{K} \alpha \lambda \dot{\alpha} \theta \eta$ mencionada por Hecateo ${ }^{7}$. $-\theta \eta$, $-\theta$ o pueden recubrir sufijos indoeuropeos muy comunes, originalmente -ta-, -to-; a su vez - $v \theta$ - de $\Sigma \alpha \imath \gamma \alpha \dot{\alpha} \nu \theta \eta \imath$, junto con el conocido 'A $\rho \gamma \alpha \nu \theta \omega \dot{v \imath \iota \varsigma}$

\footnotetext{
${ }^{6}$ En nuestro THA pp. 149 ss. y nn. 296, 298.

7 Diferentes propuestas recogidas en nuestro THA n. 296. Añadimos que Untermann, J., Monumenta linguarum Hispanicarum (en adelante $M L H$ ). Wiesbaden, 1975-1997, 4 vols., señala en vol. IV, pp. 432, 594 nombres como Calati, Calat $(i)$ us procedentes de Lusitania como paralelos de kalatokum en uno de los bronces de Botorrita, MLH IV. K.1.3.4.19.

Tal vez cuando Heródoto dice (2.33, cf. 4.49) que los

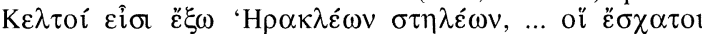

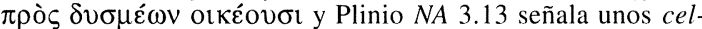
tici en la zona del Guadiana adquieren estos nombres, sobre todo $\mathrm{K} \alpha \lambda \alpha \dot{\theta} \eta$, particular sentido. Cf. también Herodoro 2a y nuestro THA pp.275 ss.
} 
Argantonio, contienen el formante también muy común -nt-; en todas estas palabras, la dental era evidentemente pronunciada de una forma particular que siglos más tarde fué mantenida por los griegos, p. ej., en el nombre de Viriato Oupí $\alpha \theta$ os tal como transcribe Estrabón 3.4.5, o Oủıpi $\alpha \theta$ os en Dión Casio 73.1 ss. ${ }^{8}$ Habría que preguntarse también que se quería representar con la $-\eta$ - de $v \imath \eta \theta \omega \iota{ }^{9}$. Si es jonio y está por - $\bar{\alpha}-$, como $\mathrm{M} \alpha \sigma \tau \imath \eta$ voí ( $F r$. 40, 42, $43,44){ }^{10}$ que exhibe Hecateo en algunas de sus secuencias auténticas, estaríamos ante un tal Niato en vez de Nieto.

3. El resto de las inscripciones andaluzas y las valencianas son particularmente interesantes ${ }^{11}$ desde el punto de vista epigráfico y dialectal pero hacen referencia a un mundo fundamentalmente helénico. Por lo tanto, sobrepasando por el momento esta importante zona, a la que volveremos más adelante, proseguiremos en la Periégesis hecateica rumbo al encuentro con los Pirineos. El grueso de los nombres que a continuación ofrece Hecateo pertenece al mundo ibero, en sentido restringido; a su vez, la mayor parte de los epígrafes griegos hispanos se concentran en Ampurias y cercanías, aunque, como hemos dicho, las referencias que en ellos aparecen desbordan ampliamente esta localización.

La gran densidad de epígrafes griegos de la zona emporitana pone de relieve cuestiones importantes en relación con el tema que nos ocupa. En primer lugar, se demuestra que en época de Hecateo miles de $\mathrm{km}$ podían ser salvados y se mantenía la cohesión entre remotas regiones gracias a la lengua griega, particularmente en dialecto jonio, del que tanto Hecateo como algunas de las inscripciones encontradas en Iberia proveen extraordinarios testimonios ${ }^{12}$. La otra cara de este fenómeno es la necesa-

${ }^{8}$ También se encuentra - $\tau \theta$ os, aunque uitiose según el editor de Dión Casio, Boissevain. El sonido no encontró grafema que lo representara en latín, solamente - $t$ - (a veces -th-) ni tampoco en las escrituras prerromanas hispánicas.

9 Para lo que cf. tal vez la forma neito del primer bronce de Botorrita $M L H$ IV, K.1.1.A.6, que en algún caso se ha relacionado con antiguo irlandés nía 'héroe': ver en $M L H$ IV, pp. $516-7$.

${ }_{10}$ Frente a M $\alpha \sigma \tau \imath \alpha v o i ́$ en Plb. 3.33.9, o en su fuente $(F r$. $41,52)$, Esteban de Bizancio. Cf. también M $\alpha \sigma \sigma \alpha \lambda \iota \eta ́ \tau \alpha$ en EGH 2.1 (Ampurias, I a.C.).

"IGAI 16, 17, 21 (EGH 11.3, 10.1, 7.1); cf. de Hoz, J., «Ensayo sobre la epigrafía griega de la Península Ibérica», Veleia, 12, 1995, 151-179, especialmente 163 ss.

12 Hecateo es recordado ( $F r .23$ ) en la Antigüedad como modelo del dialecto jonio, junto con Homero e Hiponacte; también unido a Hiponacte y Anacreonte en Fr. 196; los últimos Frs. recopilados del historiador (145, 327, 364 Mette) son todos ejemplos dialectales en ese sentido. En los epígrafes de Iberia se han descubierto rasgos fonéticos jonios (incluso con huellas eolias, IGAI 1, EGH 2.14, ver Sanmartí, E. y Santiago, R. A., «Une lettre grecque sur plomb trouvée à ria utilización pública y privada de la escritura. La manera orgullosa y consciente con la que Hecateo manifiesta $\tau \dot{\alpha} \delta \epsilon \quad \gamma \rho \alpha \dot{\phi} \omega \omega$ en su primer fragmento ${ }^{13}$ tiene su paralelo en las menciones de la propia escritura encontradas en nuestras inscripciones: $\tau \hat{\eta} \varsigma$ $\gamma \rho[\alpha \phi \hat{\eta} \varsigma$ de $I G A I 5.8$ (EGH 2.16) [450 a.C.] unido a vó $\mu$ o de la línea 7 permite desvelar la trabazón de la escritura con un impulso de institucionaliza-

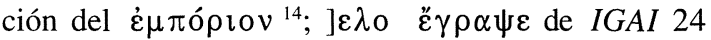
(EGH 2.41) [entre el VI y el V a.C.] posiblemente se refiere al dibujo o pintura en el vaso en el que realizó la inscripción. Un dato especialmente relevante que se deriva de varias de estas inscripciones es la gran vitalidad en esta época de la escritura portátil, la carta y el documento privado. No es raro, por lo tanto, que en ellas encontremos algunos de

Emporion», ZPE 68, 1987, [119-27], 121). Insistiremos aquí en algunos rasgos léxicos, que hasta cierto punto confirman el ámbito jonio-eolio testimoniado: $\dot{\alpha} \kappa \alpha ́ \tau$ tov de IGAI 7 (EGH 2.14) se encuentra previamente en Arquíloco 130.25 y en Alceo 305.1.8; para los antropónimos construidos a partir de hidrónimos, costumbre jonio-eolia, ver infra y n. 46. En lo que se refiere solamente al mundo jonio, la palabra $\alpha \sigma \beta o-$ $\lambda$ os (IGAI 4.3, EGH 2.56.3) está citada por primera vez en Hiponacte 185; tenemos $\dot{\alpha} \sigma \beta o ́ \lambda \eta$ en Semónides 7.61. A este último autor hay que recordar (Semon. 4) en relación con la utilización del dativo $\alpha \rho \imath \theta \mu \omega \hat{\imath}$ (IGAI 7.4, EGH 2.14.4), cf. Hdt.3.6; el sentido de $\dot{\alpha} \rho \chi \eta ́$ como autoridad o poder en IGAI 4.14 ( $E G H 2.56 .14$ ), estaría por primera vez atestiguado en Bías de Priene según Arist. EN $1130^{\mathrm{a}} 1 ; \delta i \zeta \varepsilon \sigma \theta \alpha \mathrm{l}$, como recuerda H. Rodríguez Somolinos en el comm. a IGAI 3 (EGH 2.43 ) está además de en la épica (cf. también $\dot{\rho} \rho ı n j \omega$ de IGAI 2, EGH 2.38, única cita de tal palabra en singular después de Homero) en Anacreonte 15 y en Heródoto, precisamente en 4.151 en los prolegómenos al «viaje de Coleo».

13 Dato puesto de relieve por Diels, H., «Herodot und Hekataios», Hermes, 22, 1887, 436 y n. 1, quien recuerda que Hecateo es el primero que manifiesta que «escribe» su obra. Hecateo se interesó por la historia de la escritura: dentro de una línea «milesia», mantenía que la escritura había sido traída de Egipto por Dánao ( Fr. 20). Este interés por la escritura (primer intento de análisis del fenómeno lingüístico, ver Meillet, A., «La langue et l'écriture», Scientia 26, 1919, p. 293) se complementa en Hecateo con su preocupación «etimológica», ver infra y nn. 17 ss.

14 No falta en nuestros epígrafes la terminología colonial,

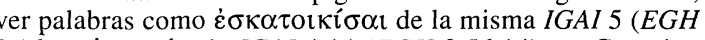
2.16) о $\dot{\alpha} \pi$ olкí $\alpha$ de IGAI 4.14 (EGH 2.56.14), en Casevitz, M., Le vocabulaire de la colonisation en grec ancien. París,

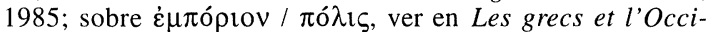
dent. Actes du colloque de la Villa Kérylos. Roma, 1991, los trabajos de Lévêque, P., "Les grecs en Occident», p. 17 y Rouillard, P., «Les emporia dans la Méditerranée Occidentale aux époques archaïque et classique», 95-108. Hay que

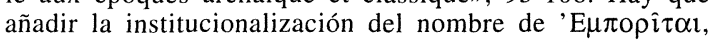
IGAI 1 (EGH 2.14) y 7 o la marca $\Delta \eta \mu$ en ladrillos de Ampurias, EGH 2.37, lo que indica su pertenencia al demos de la ciudad, cf. de Hoz, J., «Griegos e iberos: testimonios epigráficos de una cooperación mercantil», quien en Iberos y griegos: lecturas desde la diversidad. Simposio internacional celebrado en Ampurias, 3-5 de Abril, 1991. Eds. Cabrera, P., Olmos, R. y Sanmartí, E., HArq., 13 (2), 1994, 243271 , resalta también la vinculación del mundo de la emporíe con la escritura. 
los más antiguos ejemplos de $\dot{\varepsilon} \pi \imath \sigma \tau \varepsilon \dot{\varepsilon} \lambda \omega$, $\dot{\varepsilon} \pi \imath \sigma \sigma \lambda \eta \dot{~(I G A I ~ 1.12, ~ 4.13, ~ E G H ~ 2.14 .12, ~ 2.56 .13) ~}$ que se adelantarían a sus hasta ahora más antiguas menciones en Heródoto.

El hecho de la existencia de un cierto número de textos sobre plomo, no solamente epistolográficos, encontrados en Iberia y su uso también en las colonias del mar Negro dependientes de la Mileto natal de Hecateo, lleva a reflexionar sobre la particular importancia que en ese momento pudo adquirir el pesado metal en el mantenimiento del flujo de la información y de la cohesión entre metrópolis y colonias, factorías, etc., hecho que pudo coadyuvar a la realización de una obra como la de Hecateo ${ }^{15}$.

4. Hecateo no sólo reflexionó sobre la escritura, uno de los primeros análisis de componentes no semánticos de la lengua, sino que también entró en el terreno de alguno de los intentos de análisis semánticos que se ensayaban en la época, especialmente la creencia de que el nombre provee cierta información verdadera sobre elementos reales del referente ${ }^{16}$.

Es posible que la imposición o adaptación de nombres propiamente griegos a ciertas poblaciones o accidentes geográficos fuera utilizada por Hecateo (y el impulso colonizador griego) en el intento de dar unas mínimas claves para un mapa de recursos que podía ser también uno de los objetos de la $P e$ riégesis de Hecateo ${ }^{17}$. Hemos puesto antes de relie-

${ }_{15}$ Para el uso del plomo en las documentos privados, ver bibliografía en $E G H$ p. 41, de Hoz, J., «Ensayo ...», cit. (n. 11 ), p. 158,167 y n. 68; muy utilizado en los documentos iberos: de Hoz, J., «L'écriture ibérique», en Les ibères (Catálogo de exposición), Barcelona, 1997, [191-203], 198, censa setenta plomos ibéricos. En las colonias milesias del mar Negro, ver por ejemplo $n^{\circ} 23$ (donde la carta sobre plomo es llamada $\mu \mathrm{o} \lambda$ i $\beta \delta \mathrm{ov})$ y 25 de Dubois, L., Inscriptions grecques et dialectales d'Olbia du Pont. Ginebra, 1996.

${ }^{16}$ Hecateo sería contemporáneo de Teágenes de Region y otros que a partir de la semántica de los óvó $\mu \alpha \tau \alpha$, básicamente los nombres propios, cuestión que será retomada en e Cratilo de Platón, llegarán a establecer los rudimentos de la lingüística, cf. ya Untersteiner, M., Fisiologia del mito. Milán, 1946, 207 y ver en nuestro «Teorías semánticas en la Antigüedad», Introducción a la lexicografía griega. Madrid, 1997, 10 ss., 28 ss.; cf. más recientemente y en relación con Hecateo, Nicolai, R., «Pater semper ...», cit. (n. 2), 150, 154.

${ }_{17}$ Cf. Diels, H., «Herodot und Hekataios», cit. (n. 13), p. 442 ; más recientemente se ha propuesto, un tanto excesivamente, que Hecateo dirigía una misión secreta milesia buscando un lugar de emigración ante el auge de los persas, ver Moscarelli, E., «Ecateo: verifice e proposte», Atti della Accademia Pontaniana, 42, 1993, 129-146. Creemos que se trata de un fenómeno más general relacionado desde antes con la expansión colonial y comercial y a ello se deberían hechos como la imposición de los topónimos en -oussa (cf. García Bellido, A., Hispania Graeca. Barcelona, 1948, I, pp.72, 74) así como la actividad «descubridora» a la vez que de «señalización» que implica $\kappa \alpha \tau \alpha \delta \varepsilon i \kappa v v \mu \imath$, atribuida a los foceos, Hdt.1.163, o a los circunnavegantes de África, Hdt.4.42: ver ve que el plomo pudo, en cierta época, ser un soporte ideal, casi de primera necesidad para el flujo de información en el Mediterráneo: creemos que no es casual en relación con ello el que Hecateo registre

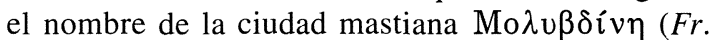
44) próxima a Málaga, con lo que muy probablemente se está dando una clave de la localización y explotación del pesado metal ${ }^{18}$. Como veremos, algunas de las inscripciones griegas que estudiamos permiten contemplar de cerca la actividad generada por la explotación de estos recursos.

5. Como hemos dicho, uno de los fines de estos breves textos transportables es la transmisión de la información. Para ello no basta con tener un soporte relativamente sólido para la escritura, sino que debe existir una organización totalmente garantizada, aunque se trate de un sistema «pre-postal», casi de fiables «mandaderos».

Insistiendo en la teoría semántico etimológica de Hecateo ${ }^{19}$, hay que decir que es llamativo el número de «etimologías», o más bien derivaciones eponímicas, que aduce nuestro historiador basándose en aspectos fundamentales de la navegación, con un tratamiento casi respetuoso, de «heroización» racionalista de la organización de la tripulación. El navegante que se aproxima, instala o muere en una región extraña, puede convertirse en «héroe epónimo» de una ciudad o de un accidente geográfico. Según Hecateo, la famosa isla de Faro (Fr. 307) en la de-

nuestro THA pp. 246-7 y n. 500. Hecateo, que fué listado por Plinio NH 1.18 junto con otros que se ocuparon de naturae frugum, ofrece nombres como el de la isla (ibera) Kpo-

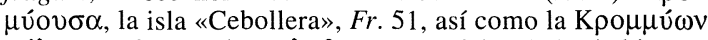
$\pi$ ó $\lambda_{1} \zeta(F r .349)$ y $\Phi \alpha \sigma \eta \lambda$ ov̂ $\sigma \sigma \alpha \mathrm{t}$ las «islas de las habichuelas» próximas a Libia ( $F r$. 353); es llamativa la curiosidad casi botánica de Hecateo por la $\alpha \kappa \alpha \nu \theta \alpha \kappa v \vee \alpha ́ \rho \alpha$ «alcachofa» en Hircania (Fr. 291) y junto con ella, la mención del sauce y tamarisco al oeste de los partos ( Fr. 292a). Cuando

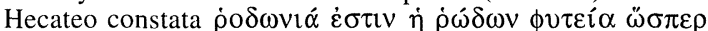
$i \omega v i \grave{\alpha} \dot{\eta} \tau \hat{\omega} v i \hat{\omega} v(F r .37)$, tal vez trataba de servirse de estos nombres como base «etimológica» de los de famosos pueblos griegos, cf. Jacoby, comm. p.329.

Lo mismo puede decirse de derivados de nombres de animales, especialmente para indicar recursos ganaderos $u$ otros, ver en nuestro THA p. 138 ss., 151 y n.304.

18 García Bellido advertía de la coincidencia de la región de los mastianos (zona costera de Murcia y Almería) con explotaciones de plomo (y plata), Hispania Graeca, cit. (n. 17), 133-4; siguiéndole Tapia Garrido, J.A., Historia general de Almería y su provincia, Almería, 1992, I, 108 ss., localiza Molibdina en Villaricos. Ver en nuestro THA pp. 149 ss. otras identificaciones y cierta bibliografía, donde seña-

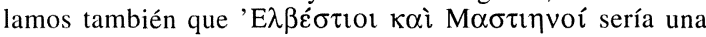
de las raras secuencias auténticas de Hecateo y que si Mo$\lambda v \beta \delta i v \eta$ es «la del plomo», en el nombre 'E $\lambda \beta \varepsilon ́ \sigma \tau \imath$ o habría tal vez alusión a la plata, cf. también id. THA pp. 6-11; el oro y la plata son mencionados en el Fr. 45 Nenci hecateico sobre "I $\beta v \lambda \lambda \alpha$. Experiencias metalúrgicas en IGAI 4 (EGH 2.56)

${ }^{19}$ Cf. supra y n. 16. 
sembocadura del Nilo se llama así por el jefe de proa o pilotín de Menelao; Canobo o Canopo ( $F r$. 308) en la misma zona, por el piloto del mismo héroe homérico, cuya llegada a la isla de Faro es narrada en $O d .4 .355 \mathrm{ss.}{ }^{20} \mathrm{El}$ sistema interpretativo no se limita forzosamente al mundo griego: estamos en un momento en el que lo no griego todavía no es tajantemente «bárbaro» y hay mucho que aprender de ello ${ }^{21}$. Así, según Hecateo, la ciudad de Nagido ( $F r$. 266) y la isla Nagidusa (a medio camino entre Cilicia y Panfilia) reciben su nombre del piloto, evidentemente no griego, Nágide. Esta autoridad extraordinaria y trascendente de los oficiales del barco tiene su reflejo en nuestras inscripciones: en IGAI 4 (EGH 2.56) se menciona a un tal Tibeco, de nombre evidentemente bárbaro, al que se confía una carta; es «amigo del piloto» y con influencia «en la colonia»; más adelante volveremos sobre esta tablilla. La existencia de un «correo» de cartas y notas, basado en la confianza en los oficiales del barco, abre la posibilidad a una corriente de textos mas elaborados ${ }^{22}$ mediante los que un investigador como Hecateo podía mantenerse bien informado.

6. La «eponimización» del mundo naútico en Hecateo no se detiene en los oficiales del barco: es curioso que no se le pasara desapercibida la vital importancia del diseño náutico y curiosamente, indígena, local. Así, sugiere que el nombre de unos ligeros pero prácticos esquifes, los $\Lambda_{\imath} \beta \nu \rho v \imath \kappa \dot{\alpha} \sigma \kappa \alpha ́ \phi \eta$,

${ }_{20}$ Ello implica la teoría de que los viajes de Menelao reseñados en varios puntos del canto IV de la Odisea comportan una «explicación» geográfica, a base de epónimos de localidades visitadas: cf Jacoby comm. p.369; sobre la relación de Hecateo y la épica, ver últimamente Nicolai, «Pater semper ...», cit. (n. 2), 150 y n. 19. Recordemos que los comentaristas antiguos dieron importancia a los viajes de Menelao (los gramáticos Aristónico y Crates de Malo, el historiador Polibio), llegando a pensar que se prolongaron más allá de Gibraltar: ver en nuestro THA pp. $30 \mathrm{ss}$.

21 Nenci, G., «La filobarbarie di Ecateo nel giudizio di Eraclito», Rivista di filologia classica, 27, 1949, 107-117; la aproximación a los indígenas es una constante de los establecimientos griegos desde Huelva a Béziers, según Mohen, J.P., Rouillard, P., Éluère, C. «Les ibères et leurs contemporains», en Les ibères, cit. (n. 15), p.42; ver en Les grecs et l'Occident, cit. (n. 14), los trabajos de De la Genière, J., «Les grecs et les autres. Quelques aspects de leurs relations en Italie du Sud à l' époque archaïque», 29-40 y de Morel, J.P., «Les grecs et la Gaule», 41-69, quien resalta el «comercio de técnicas» entre Marsella y los pueblos indígenas (p. 63): tal vez en época de Hecateo desde el mundo no griego podían ser provistas todavía algunas técnicas, ver infra la búsqueda autónoma de soluciones técnicas en IGAI 4 (EGH 2.56) y en relación con los etruscos.

${ }_{22}$ Cf. $\mu \nu \eta \mu o ́ \sigma v v \alpha \pi \alpha \dot{\alpha} \nu \omega \nu \gamma \rho \alpha \dot{\phi} \varepsilon \sigma \theta \alpha \mathrm{l}$ referido a la memoria escrita que se atribuye al propio Homero en Vida de Homero 6 del Ps. Heródoto, cuando se hace al poeta gran viajero (también por Iberia y Etruria) queriéndose crear evidentemente el paradigma del curioso viajero y del «historiador». Volveremos sobre este texto, para el que cf. también nuestro THA pp. 2-5. proceden del de «cierto» personaje llamado Liburno (Fr. 93). Este aprecio por las naves pequeñas y ligeras, complementarias de las grandes y del que encontramos importantes menciones en algunas de nuestras inscripciones, tiene precedentes antiguos. Hesíodo presenta el primero la oposición y complementariedad de la «nave pequeña» con la «nave grande». Si bien contempla la «nave pequeña»

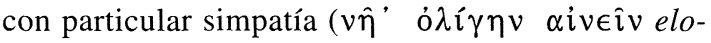
giar la nave pequeña, Op. 643), también opina que, si hay que arriesgarse, es mejor hacerlo en gran escala y poner las mercancías en una «nave grande», lo que implica la existencia de armadores que reunan carga de varias procedencias y redes comerciales que garanticen que a mayor carga, multiplicación del

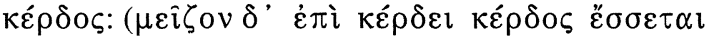
mayor ganancia se añadirá a la ganancia, Op. 644$5)^{23}$. En época casi contemporánea de Hecateo, los plomos de Ampurias IGAI 1 (EGH 2.14) y de PechMaho IGAI 7, evidencian todavía la vigencia de la mencionada oposición hesiódica trasladada a la hormigueante actividad colonial, unida a la necesidad del trato con naturales de la zona. El autor, probablemente un armador, de $I G A I 1$ (EGH 2.14), igual que

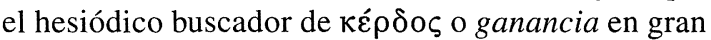
escala con la «nave grande», parece exigir un mínimo de unidades o medidas, en número de veinte o diez de vino ${ }^{24}$, sin el que la $\dot{\varepsilon} \mu \pi$ opí no resultaría rentable. Ahora bien, en lugares de escasas instalaciones portuarias, es forzosa la utilización de una serie de barcas (lanchas, chalanas) de reducidas dimensiones capaces de cargar y descargar transbordando de y a la «nave grande» fondeada a cierta distancia. Esta «nave pequeña» podría estar reflejada en

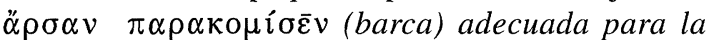
navegación costera de IGAI 1 (EGH 2.14) y en los $\dot{\alpha} \kappa \alpha ́ \tau \imath \alpha$ las barcas $^{25}$ de IGAI 7, que, sintomática-

${ }^{23}$ En la antinomia «nave pequeña / nave grande» descubre Musti, D., L'economia in Grecia. Bari, 1981, 34 ss., uno de los ejemplos de la polaridad fundamental de la economía griega. La «nave pequeña» de Hesíodo sería un simple bote, según Gray, D., Archaeologia homerica. Die Denkmäler und das frïhgriechische Epos, Gotinga, 1974, 136.

${ }_{24}$ En el universo «eponímico-etimológico» de Hecateo la colonia milesia de Sinope recibe su nombre de un término bárbaro minorasiático en relación con la «ebriedad»: ver infra y n. 27. Hecateo reseña también una ciudad de nombre

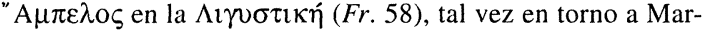
sella; sobre los egipcios como bebedores de vino, ver el $\mathrm{Fr}$. 338 Nenci.

25 Santiago, R.A. y Sanmartí, E., «Notes additionnelles sur la lettre sur plomb d'Emporion", $Z P E 72,1988$, p. 100 y n. 3; Santiago, R.A., «Quelques corrections à $Z P E$ 80, 1990, pp. 79-80», ZPE, 84, 1990, p. 14; van Effenterre, H. y Ruzé, F. Nomima, II, Roma, 1995, pp. 268 ss.; cf. de Hoz, J., «Los negocios del Sr. Heronoiyos» (en prensa); de todas formas, para $\ddot{\alpha} \rho \sigma \alpha \nu$, ver infra n. 28; para los $\dot{\alpha} \kappa \alpha \dot{\tau} \tau \alpha$, también supra n. 12. 
mente, están fondeadas en el río ( $\pi$ o $\tau \alpha \mu$ ó $\varsigma)$ desde donde es posible introducirse tierra adentro. Nuestros IGAI 1 ( $E G H 2.14$ ) y 7 muestran que en un lugar muy alejado de las metrópolis helenas había forzosamente gran demanda de estas pequeñas embarcaciones, bien adaptadas al medio, y de sus expertos patrones locales: asistimos a un probable acaparamiento de «barcas» ( $\dot{\alpha} \kappa \alpha \dot{\tau} \tau \imath \alpha$ en IGAI 7 y $\ddot{\alpha} \rho \sigma \alpha \nu \pi \alpha \rho \alpha \kappa o \mu \imath \bar{\varepsilon} \bar{\varepsilon} v$ de IGAI 1.5-13, EGH 2.14.513) y en esta última inscripción a la captación de personas expertas (si hubiese dos, que envíe a los dos) a las que se ofrece ventajosa asociación con tal de conseguir este transporte $(\tau \hat{\omega}] \mu \nu \sigma u \quad \mu \varepsilon \tau \varepsilon \dot{\varepsilon} \varepsilon \tau \omega$ que participe con la mitad, 1. 11); todo debe hacerse en el menor tiempo posible $\dot{\omega} \varsigma \alpha \ddot{\nu} \delta$ $\delta \dot{v} \eta \tau \alpha \iota$ $\tau \alpha ́ \chi \imath \sigma \tau \alpha$; recordemos también las participaciones, anticipos, señales y fianzas para adelantarse y conseguir estas lanchas en IGAI 7; también en IGAI 8, aunque no sabemos sobre qué versa, se ofrece probablemente un trato ventajoso. Como vemos, todo ello obliga forzosamente al trato, casi dependencia de los habitantes de la zona: Bóo $\pi \varepsilon \delta$ [ de IGAI 1.4, 7 (EGH 2.14.1, 7) auténtico agente del autor de la carta y $\mathrm{B} \alpha \sigma \iota \gamma \varepsilon \rho \rho \circ \varsigma$ uno de los testigos del complejísimo trato de IGAI 7 tienen nombres ibéricos ${ }^{26}$. Todo ello pone de relieve fragmentos de la realidad que Hecateo consideró digna de «eponimización» en la figura del adriático Liburno.

7. Hemos visto que el nombre de la ciudad

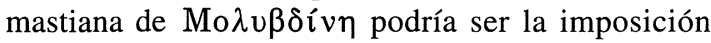
de un nombre griego con la intención de señalar ciertas características relevantes locales; también, que Hecateo transmite una serie de nombres étnicos y de lugares geográficos indígenas que pueden ser adaptaciones al griego, ilustrando este caso con el nombre de la ciudad de $K \alpha \lambda \alpha \dot{\alpha} \theta \eta$, probablemente en el sudoeste de Andalucía. Prosiguiendo ahora la línea de exposición de la Periégesis, ya en el Levante

${ }^{26}$ Sobre B $\alpha \sigma \pi \varepsilon \delta[$, ver de Hoz, J., en Sanmartí, E. y Santiago, R.A., «Une lettre grecque sur plomb ...», cit. (n. 12), 123; de Hoz, J., «La lengua y la escritura ibéricas, y las lenguas de los íberos», Lengua y cultura en la Hispania prerromana. Eds. J. Untermann y F. Villar, Salamanca, 1989, 654 Velaza, J., «B $\alpha \sigma \pi \varepsilon \delta$ - sur le plomb grec d'Emporion: un anthroponyme ibérique?», $B N, 27,1992,264-267 . \mathrm{B} \alpha \sigma \mathrm{l}$ $\gamma \varepsilon \rho \rho \varsigma_{\varsigma}$ sería la transcripción griega de un ibérico basi-keŕe ver de Hoz, J., «La lengua y la escritura ibéricas ...», cit., 654; id., «Griegos e iberos: testimonios epigráficos de una cooperación mercantil ...», en Iberos y griegos... Eds. Cabrera, P., Olmos, R. y Sanmartí, E., HArq. cit. (n. 14), 243-271, pone de relieve que al menos una parte del trayecto comercial estaba en manos iberas. Sobre esta navegación fluvial o en zonas pantanosas con barcas autóctonas, complementaria del gran barco comercial, ver en relación con el plomo de Pech Maho, van Effenterre, H. y Ruzé, F., Nomima, II, Roma, 1995, 268 ss.; tales terrenos pantanosos conforman «paysages phocéens typiques», según Morel, J., «Les grecs et la Gaule», Les grecs et l'Occident, cit. (n. 14), 52.
Peninsular, nos encontramos con la ciudad de $\Sigma \iota \kappa \alpha ́ v \eta \cdot \pi o ́ \lambda \iota \varsigma$ 'I $\beta \eta \rho i \alpha \varsigma$ (Fr. 45).

En el trasfondo de la «eponimia» y «etimología» de Hecateo se encuentran mitos que formarían el primer entramado que «explicaría», como ya hemos visto, nombres de pueblos y ciudades. En algunos casos, cuando el autor no acaba de encontrar la evidencia de la relación «etimológica», considera que se ha producido un proceso de corrupción $(\kappa \alpha \tau \grave{\alpha}$ $\phi \theta o \rho \alpha ́ v)$, casi de «cambio lingüístico» en el que él decide intervenir y si es necesario forzar una etimología, incluso introduciendo un mito desconocido hasta el momento. Por ejemplo, según nuestro historiador $(F r .34)$ el nombre de la ciudad de $\Sigma \imath \nu \omega ́ \pi \eta$ procede de la «corrupción» de la palabra $\sigma \alpha \nu \alpha \dot{\pi} \pi \alpha$ l ebrios, en el «dialecto» de las Amazonas y en relación con un mito que haría referencia a una de és$\operatorname{tas}^{27}$.

Se ha propuesto para Saigante o Seganta de IGAI 1 (EGH 2.14) una identificación con Sagunto ${ }^{28}$ : consideramos que la hecateica y enigmática Sicane debiera ser tomada en consideración en relación con esta interpretación. Pensamos que lo mismo que, como acabamos de exponer, Hecateo explica $\Sigma \imath v \omega ́ \pi \eta$ como una «corrupción» de $\sigma \alpha \nu \alpha \dot{\pi} \alpha$, cabe la hipótesis de que adaptara $\Sigma \alpha i \gamma \alpha \nu \theta \alpha$ o

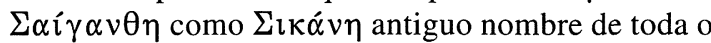
parte de Sicilia, basándose en alguna afinidad lingüística aparente o real: no deja de ser curioso que en una inscripción ibérica de Sagunto aparezca sike-

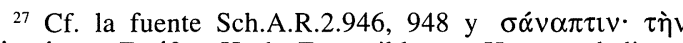
oivı $\omega \tau \eta \nu, \Sigma \kappa u ́ \theta \alpha \imath$ Hsch. Es posible que Hecateo dedicara cierta atención a esta explicación mítico-lingüística del nombre de Sinope en el mar Negro, ciudad con vínculos coloniales con Mileto y en cuyas inscripciones no faltan personajes con el nombre 'Eк $\alpha \tau \alpha$ îos: ver n's 1, 2, 102 de Dubois, Ins criptions grecques dialectales ..., cit. (n. 15). Hecateo acude con cierta frecuencia a este proceso de «cambio lingüístico»:

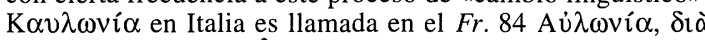

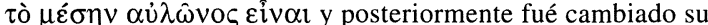
nombre a Caulonia; en el mismo $F r$. nos informa que 'E $\pi \hat{i} \delta \alpha \cup \rho \circ \varsigma$ procede de 'E $\pi i \tau \alpha v \rho \circ \varsigma, \mathrm{K} \lambda \alpha \zeta o \mu \varepsilon v \alpha i$ de

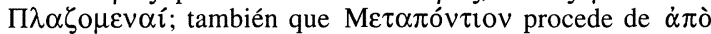

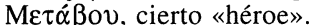

${ }_{28}$ J.L. Melena, en Santiago, R. A. y Sanmartí, E., «Notes additionnelles ...», cit. (n. 25), 100 y n. 1; la forma Zá $\alpha \alpha v \theta \alpha$ (con variantes) sería una «réfaction», un «cultismo» interesa-

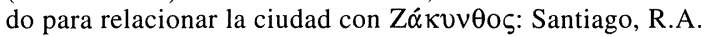
«Encore une fois sur la lettre sur plomb d'Emporion», $Z P E$, 80, 1990, 79-80, id., «En torno a los nombres de Sagunto», Saguntum 23, 1990, 123-140. No queremos pasar por alto la propuesta de López García, A., «Nota sulla lettera di piombo da Emporion», Tyche 10,1995 , donde se propone para la secuencia $\alpha \rho \sigma \alpha \nu$ de $I G A I 1.5$ (EGH 2.14.5), el nombre de la ciudad "A $\rho \alpha$ : cabe una posibilidad de que, en lugar de una ciudad situada vagamente en el sur de la Península, estemos aquí ante una versión de arse, el nombre ibérico de Sagunto, según conocemos por las más antiguas leyendas monetales hispánicas, ver García-Bellido, M.P. y Ripollès, P.P., «Prestige et espace économique des ibères», en Les ibères, cit. (n. 15), pp. 205-215, p. 207 
$u n^{29}$. A su vez, los precedentes de la explicación «histórica» del primitivo poblamiento de Sicania o Sicilia por parte de iberos procedentes del río Sicano que algunos historiadores antiguos de relieve consideraron perfectamente «científica», podrían haberse iniciado en alguna explicación etimológica enunciada por Hecateo ${ }^{30}$.

En el avance de sur a norte ${ }^{31}$ de la Periégesis de Hecateo, encontramos $\mathrm{K} \rho \alpha \beta \alpha \sigma i \alpha \cdot \pi$ ó $\lambda \iota \varsigma$ 'I $\beta \eta ́ \rho \omega \nu$ (Fr. 46). En otra ocasión habíamos propuesto ${ }^{32}$ que tal vez en el nombre de esta ciudad ibérica, $-\beta \alpha \sigma$ - podría ser un elemento que aparece con frecuencia en la onomástica propiamente ibérica y concretamente en los mencionados Bá $\sigma \pi \varepsilon \delta[$

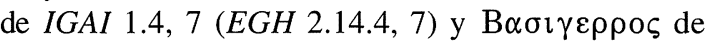
IGAI $7^{33}$. Sin embargo, en la medida que nos permite nuestro conocimiento particular, sólo tangencial, de las lenguas prerromanas de Hispania, consideramos que habría que dejar ese formante como lo que parece que es, algo propio de antropónimos y acudir a otros ámbitos donde puede hallarse algo más aceptable para $\mathrm{K} \rho \alpha \beta \alpha \sigma i \alpha^{34}$.

${ }^{29}$ Probablemente un nombre: $M L H$ III 2, F.11.6; cf. también sikebones G.1.6 (Alcoy), sekenius F.9.5 (Orleyl), cf. F.7.2; en un plomo ibérico del mismo Ampurias $M L H$ III 2 , C.1.6 encontramos nombres como siken, sikounin; de forma congruente con la formación con $-v \theta$ - de $\Sigma \alpha \imath \gamma \alpha \dot{v} v \theta \eta \imath$ (y ver supra y nn. $6 \mathrm{ss}$.), hay buenos paralelos en textos puramente celtibéricos: el nombre Sekontios se repite cuatro veces en el segundo bronce de Botorrita $M L H$ IV, K.1: en la línea 48 de la columna II justamente a continuación de una tal Sikeia, siendo el primer nombre de la misma columna Sekanos. Jacob, P., «Textes concernant Sagonte», Homenatge a A. Chabret 1888-1988. Valencia, 1989, 15-28, propone Sagunto como nombre celtibérico; duda Santiago, R. A., «En torno a los nombres de Sagunto», cit. (n. 28), 123, aunque en p. 131, constata autores griegos que transcriben el nombre de la ciudad y sus étnicos como $\Sigma \varepsilon \gamma$-. Formas que comienzan por $s e c-$ (seg-) en antropónimos y topónimos aparecen en zona celtibérica, pero también en el este, Untermann, J., Elementos de un atlas antroponímico de la Hispania antigua. Madrid, 1965, mapa 67. Cf. nuestro THA n.310.

${ }^{30}$ Cf. Helánico 79a, Tucídides 6.2, Filisto 45, Éforo 136, Antíoco el historiador 4, Servio Aen. 8.322-333, Avieno Ora 479-480. Ver nuestro «La Península ibérica en la tradición homérica», Actas del VII Congreso de la sociedad española de estudios clásicos. Madrid, 1989, III, 103-109 e id. THA pp. 260 ss., n. 310; también en THA I, p.137, 143, donde se recogen diferentes identificaciones de Sicane.

${ }_{31}$ Cf. Avieno Ora 479-480 attollit inde se Sicana ciuitas,/ propinquo ab amni sic vocata Hibericis (...) (489-491) post Crabrasiae iugum / procedit alte, ac nuda lit[t]orum iacent / ad usque cassae $\langle C>$ herronesi terminos.

32 En THA p. 153 y n.311. Jacob, P., «Notes sur la toponymie grecque de la côte méditerranéenne de l'Espagne antique», Ktema, 10, 1985, 263 e id. "Textes concernant Sagonte», cit. (n. 29), 15, propugna la identificación de K $\rho \alpha \beta \alpha \sigma i \alpha$ con Sagunto, siguiendo a Schulten, A. y AA.VV., Fontes Hispaniae antiquae, Barcelona, 1954-, I, p.120; duda Santiago, R.A., «En torno a los nombres de Sagunto», cit. (n. 28), 125.

33 Ver supra y n. 26.

${ }^{34}$ Eugenio Luján nos sugiere una posible relación con karbika.
Siguiendo la ruta de la Periégesis, encontramos mencionados una serie de pueblos: nos detendremos

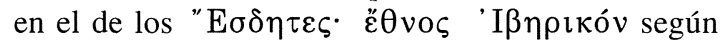
el Fr. 47 de Hecateo. Salvando ciertas dificultades, sería el etnónimo de un pueblo identificable en otras transcripciones con los sedetanos ${ }^{35}$, testimoniado a su vez en leyendas monetales ibéricas como seteisken, (y seteis, setei) ${ }^{36}$, adaptado al griego con un alargamiento en $-t{ }^{37}$. En relación con este etnónimo y con los "E $\sigma \delta \eta \tau \varepsilon \varsigma$ documentados en Hecateo habría que poner el nombre de $\Sigma \varepsilon \delta \varepsilon \gamma \omega \nu$, uno de los testigos que asisten al trato descrito en el plomo de Pech-Maho, IGAI 7, y paralelo, con las salvedades

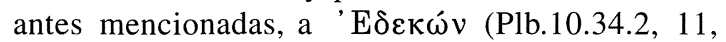
40.3), «dinasta» que consigue que los habitantes de la margen izquierda del Ebro se pasen a los romanos ${ }^{38}$.

${ }^{35}$ En los manuscritos aparece $\varepsilon i \delta \eta \tau \varepsilon ́ \varsigma$ pero la alfabetiza-

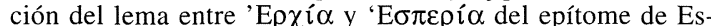
teban de Bizancio, autor que sigue el orden alfabético con rigor, hace plausible la corrección "E $\sigma \delta-$, o "E $\sigma \delta-$. Esta última lectura, que el editor Meineke piensa que apunta a los sedetanos, denunciaría la pérdida de $\sigma$-inicial, posición en la que dicho sonido no es representado a veces en transcripciones de pueblos y ciudades de la Península: aunque encontra-

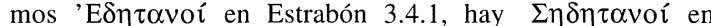
3.4.14, Sedetana cohors en Silio Itálico 3.372, cf. Tito Livio

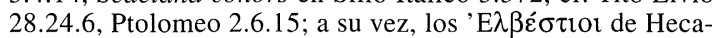
teo $\mathrm{Fr}$. 40, son llamados 'E $\lambda \beta v \sigma i v i o l$ por Herodoro. 2 a y tenemos los regna Selbyssina en Avieno Ora 42; $\Sigma \imath \hat{\xi}$ o $\varsigma_{\text {(Heca- }}$ teo Fr. 43), Ateneo 121a $\Sigma \varepsilon \xi i \tau \alpha \nu i \alpha$, Ptolomeo 2.4.7 $\Sigma \varepsilon ́ \xi$, pero en Estrabón 3.4.2 encontramos $\dot{\eta}\left(\pi \delta \lambda_{\imath} \varsigma\right) \tau \hat{\omega} v{ }^{\prime} \mathrm{E} \xi_{1-}$ $\tau \alpha \nu \hat{\omega} \nu$ y en Mela 2.94, Ex. Se trata de un fenómeno que todavía reclama una explicación general: cf. nuestro THA pp. 150 ss., nn. 300, 315 .

${ }^{36} M L H$ I.1, A 25. El morfema - $(s / n)$ ken equivaldría a sufijos de etnónimos de la tradición griega y latina del tipo -etes, -etani y sería paralelo a EMPORITSN de las monedas emporitanas: ver en general, Faust, M., Die antiken Einwohnernamen und Völkernamen auf -itani, -etani, cit. (n. 37), 32 ss., Untermann, J., «Etnónimos y lenguas de la Hispania antigua», Paleoetnología de la Península ibérica. Eds. M. Almagro Gorbea y G. Ruiz Zapatero, Complutum 2-3, 25 30; de Hoz, J., «La lengua y la escritura ibéricas», cit. (n 26), 647; Correa, J.A., «La lengua ibérica», RevEspLing, 24, 1994, 264-287; Villar, F., «Las lenguas de la Hispania prerromana», Nova Tellus, 14, 1996 [153-188], 157-158.

${ }^{37}$ La formación "E $\sigma \delta \eta \tau \varepsilon \varsigma$ como Kúv $\eta \tau \varepsilon \varsigma$ de Heródoto 2.33 y Herodoro $2 \mathrm{a}$, donde encontramos los también occidentales $\Gamma \lambda \hat{\eta} \tau \varepsilon \varsigma$, puede haber sido aplicado sobre una raíz indígena «pour constituer une flexion plus commode», Chantraine, P., La formation des noms en grec ancien. París, 1933, 265-7, ver también Faust, M., Die antiken Einwohnernamen un Völkernamen auf -itani, -etani. Gotinga, 1966, 30 ss., Fatás, G., La Sedetania. Zaragoza, 1973, 81, Tovar, A., «Notas linguísticas sobre monedas ibéricas», Actas del II coloquio sobre lenguas y culturas prerromanas de la Península Ibérica, Tubinga 1976). Eds. A. Tovar, M. Faust, F. Fisher y M. Koch, Salamanca, 1979, 486; el problema edetanos / sedetanos todavía no está resuelto, Plácido, D. «Les Ibères dans les textes anciens», en Les ibères cit. (n. 15), p. 50. Cf. p. 154 ss. y nn. 315,316 .

38 Transcrito en latín como Edesco ... clarus inter duces Hispanos por Tito Livio 27.17.1. $\Sigma \varepsilon \delta \varepsilon \gamma \omega v$ junto con 
8. Pech-Maho se encuentra en lo que fué el territorio de los elísicos, pueblo ligur según Hecateo

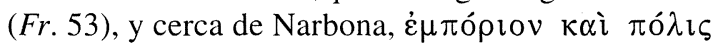

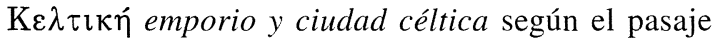
de Esteban de Bizancio que contiene el de Hecateo en el que llama a los habitantes de esta ciudad $\mathrm{N} \alpha \rho \beta \alpha$ ious (Fr. 54). Estos y otros, aunque escuetos, fragmentos de Hecateo permiten atisbar una gran variedad de pueblos próximos entre sí, (iberoselísicos-ligures) ${ }^{39}$ sobre los que el elemento griego ejerce una decisiva influencia: todo ello nos lleva a otro mínimo fragmento de Hecateo, el que mencio-

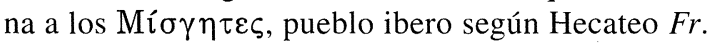
$50{ }^{40}$. Esta diversidad étnica trabada por el trato comercial autorizado en la lengua griega, se hace perfectamente patente en el mencionado plomo de Pech-Maho, IGAI 7. Tenemos al eventual griego 'Howvollos y los emporitas, en principio del

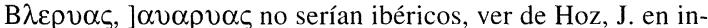
tervención en la discusión sobre el plomo de Pech-Maho en Iberos y griegos ..., Eds. Cabrera, P., Olmos, R. y Sanmartí, E., HArq. cit. (n. 14), (1), 212-3, ibid. (2), 247, aunque en «Los negocios del señor Heronoiyos», cit. (n. 25), (cf. Untermann, J., «Repertorio antroponímico ibérico», Archivo de Prehistoria Levantina, 1987, 313) considera que el monosílabo kon puede entrar en la antroponimia ibera. Ver infra y n. 43.

39 Hecateo dedicó cierta atención a los elísicos y los ligures evidenciando una relación con el mundo celta (Frs. 5358 ), cf. también Jacoby comm. pp. 332-333. Una sombra de estos fragmentos estaría en Avieno Ora, quien tras el paso por los Pirineos y las tierras sordicenas señala la gens Elesycum en los vv. 586 ss. y Nar $<b>0$ ciuitas su capital; en el v. 611 (tras una lacuna) indica que el río Orano (el Hérault) marca la frontera entre Hibera tellus y los ligures. Por su parte, Heródoto 7.165 inclûye a los elísicos, junto con iberos y ligures, luchando juntos en la batalla de Hímera, en una época que coincidiría con el acmé de Hecateo, momento en el se hace más densa la relación entre el mundo ibero y el de los elísicos, ver Solier, Y., «La culture ibéro-languedocienne aux VI-V siècles», Ampurias, 38/40, 1976/8, 211 -264. Por lo demás, pueblo mal conocido, Schulten, Fontes ..., cit. (n. 32), I, 189; II, 22-23, 68; de Hoz, J., «Ensayo ...», cit. (n. 11), 165 ss. y n. 62; id. «Los negocios del Sr. Heronoiyos», cit. (n. 25), La epigrafía de la zona de Narbona refleja la complejidad de las poblaciones de la zona, ver Untermann, J., «Lengua ibérica y lengua gala en la Galia narbonensis», Archivo de prehistoria levantina, 12, 1969, 99-116; Correa, J.A., "Antropónimos galos y ligures en inscripciones ibéricas», Studia paleohispanica et indogermanica J. Untermann ab amicis hispanicis oblata. Eds. J.A. Adiego, J. Siles, J. Velaza, Barcelona, 1993, 101-116.

El contacto entre ligures y elementos procedentes de la Península no debió ser siempre pacífico, como indica el Fr.199 de Esquilo o el mito de los sicanos / iberos huyendo de los ligures según Tucídides y otros historiadores: ver supra y n. 30, y nuestro THA pp. 197-201, 260 ss.

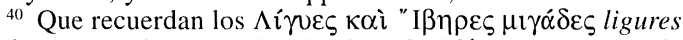

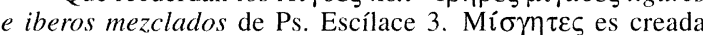
con alargamiento en $-t$ a partir de una forma jonia de la raíz de $\mu \varepsilon(i ́) \gamma v v \mu 1, \mu i \sigma \gamma \omega$, verbo este último que se encuentra en una de las escasas frases literales conservadas de Hecateo (Fr. 21). Cf. nuestro THA pp. 155, 199 ss. y n. 405.

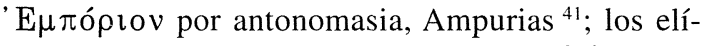
sicos o ligures estarían representados por $\mathrm{B} \lambda \varepsilon \rho v \alpha \varsigma$, ] $\alpha \cup \alpha \rho \cup \alpha \varsigma ;$ los iberos por $\mathrm{B} \alpha \sigma \imath \gamma \varepsilon \rho \rho \circ \varsigma$, del que ya hemos hablado, y por $\Gamma$ o $\lambda$ o[.] $\beta \imath$ u y $N \alpha \lambda \beta \varepsilon[..] v^{42}$.

9. La ruta alguna vez marcada por $\mu \varepsilon \tau \dot{\alpha} \quad \delta \varepsilon \ldots$ $\mu \varepsilon \tau \alpha \dot{\alpha} \delta \varepsilon$, nos acerca a Marsella o M $\alpha \sigma \sigma \alpha \lambda i \alpha$ : Hecateo ( $F r$. 55) sería el autor más antiguo que la men-

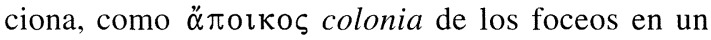
entorno ligur y céltico ${ }^{43}$. Aunque desde el punto de vista arqueológico se advierte hoy día más que supeditación de Emporion a Marsella, cierto paralelismo a finales del VI, en las inscripciones emporitanas se evidencia un innegable peso marsellés ${ }^{44}$ :

${ }^{41}$ Cuya formación en - $t$ - a pesar del cúmulo añadido de sufijos se reconoce en las formas romances cat. empordanesos, cast. ampurdaneses. Pero recordemos también que en el

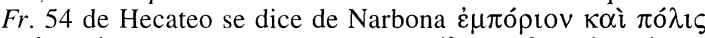

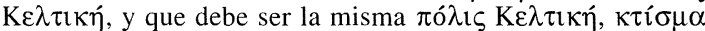
M $\alpha \sigma \sigma \alpha \lambda \iota \omega \tau \hat{\omega} v$ según St.Byz. s. $u$. 'E $\mu \pi$ óprov y a cuyo

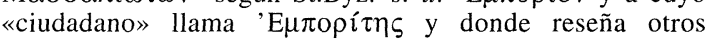
'Euлópı: ver Roman, D. y Y., Histoire de la Gaule (VI a.J. C.-Ier s. ap. J.S). Une confrontation culturelle, Paris, 1997, 251-252. Un importante documento sobre la reglamentación de las actividades de ciertos $\dot{\varepsilon} \mu \pi$ opî $\tau \alpha$ en lo que hoy es el interior de Bulgaria, en Velkov, V. y Domaradzka, L., «Kotys I (383/2-359) et l'emporion de Pistiros en Thrace», $B C H$, $118,1994,1-15$. Un intento importante de precisar el emporion aunando fuentes literarias y arqueología en Rouillard, P., «Les emporia dans la Méditerranée occidentale aux époques archaïque et classique», Les grecs et l'Occident, cit. (n. 14), 95-108.

${ }_{42}$ Sobre $\Sigma \varepsilon \delta \varepsilon \gamma \omega v$ ver supra y n. 39; cf. también De Hoz, J., «Los negocios del Sr. Heronoiyos», cit. (n. 25), quien opina (n. 37) que sólo «entrando en un auténtico juego de equivalencias arbitrarias» podríamos pensar que -v $\alpha \varsigma$ de $\mathrm{B} \lambda \varepsilon \rho v \alpha \varsigma$, , $\alpha v \alpha \rho v \alpha \varsigma$ corresponde al ibero $\beta \alpha \varsigma$; de Faria, A.M., «Epigrafia monetaria meridional», Conimbriga 30, 1991 , p. 18 cree ver en $\mathrm{B} \lambda \varepsilon \rho v \alpha \varsigma$ una mala lectura por E $\lambda \varepsilon \rho \cup \alpha \varsigma$ documentado como Eler Bas en Elne, a medio camino entre Sigean y Ampurias; id., «Subsidios para o stado da antroponimia iberica», Vipasca, 3, 1994, 69.

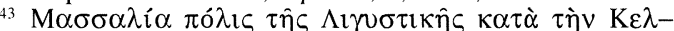

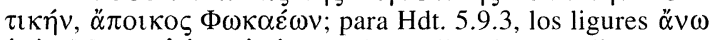

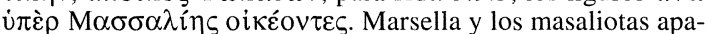
recen con frecuencia, pero dispersos, en la epigrafía («Massilian diaspora» $S E G$ 42.969), y en documentaciones no tan antiguas (dudas sobre la fecha de ] $\sigma \sigma \alpha \lambda[$ en el «Tesoro de los masaliotas» de Delfos, SEG 17.244) como la hecateica. F. Jacoby no desdeña el papel de relatos como el de Eutímenes de Masalia en la obra de Hecateo: «Über die Entwicklung der griechischen Historiographie», Klio, 1909, 83 y n. 2 .

${ }^{44}$ Sanmartí, E. y Santiago, R.A., «La lettre grecque d'Emporion et son contexte archéologique», RAN, 21, 1988, 3-17; también Rouillard, P., «Les emporia ...», Les grecs et l'Occident, cit. (n. 14), 102, quien cita los plomos de Ampurias y Pech-Maho; desde el punto de vista numismático, García-Bellido, $\mathrm{M}^{\mathrm{a}} \mathrm{Paz}$, «Las relaciones económicas entre Massalia, Emporion y Gades a través de la moneda», Iberos y griegos..., Eds. Cabrera, P., Olmos, R. y Sanmartí, E., HArq., cit. (n. 14), (2) 117-149; Sanmartí, E., «Emporion, port grec à vocation ibérique», La Magna Grecia e il lontano occidente. Atti del XXIX convegno di Studi sulla Magna Grecia (Taranto 6-11 Ottobre 1989). Tarento, 1990, 389-410 constata la fuerte relación entre Marsella y Ampurias a mediados del 


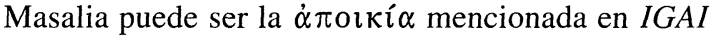
4.15 ( $E G H 2.56 .15)$ y todavía en el siglo I, en una inscripción emporitana en texto jonizante ( $E G H$ 2.1 ), encontramos personajes que se dicen masaliotas. Se mantiene, además, en la antigua Ampurias una onomástica basada en hidrónimos de Asia Me-

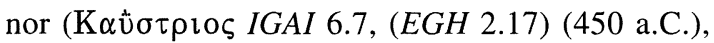

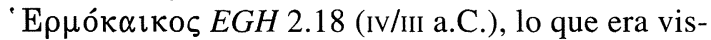
to en la Antigüedad como típico de Masalia ${ }^{45}$.

10. La ruta marcada por Hecateo pasa de Marsella a Etruria. Las dos caras de la relación entre ambas en esta época ${ }^{46}$ se reflejan, como en una moneda, en el plomo de Pech Maho, IGAI 7, cuyo autor, aunque sólo reciclara una de las caras de la lámina de plomo, actuó como eslabón entre el tráfico etrusco, el griego y la península ibérica: la cara $\mathrm{A}$, en etrusco, lleva escrito el nombre de Marsella ${ }^{47}$; en la cara B, en griego, aparecen los emporitas, teniendo como telón de fondo un mundo indígena diverso, del que ya hemos hablado.

VI a. C. y su decadencia a partir del v. Es posible que en época próxima a Hecateo, incluso influenciado por él, se produzca la aseveración de Esquilo Fr. 73a, de que el Erídano, identificado con el Ródano, está en Iberia, es decir, advierte la prolongación de una franja de influencia ibérica casi hasta Marsella, cf. los, (según Schulten, Fontes ..., cit., (n. 32), I, 189) Laterculi Alexandrini (= PBerol.inv.13044) que toda-

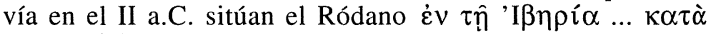
M $\alpha \sigma \sigma \alpha \lambda i \alpha v$. Cf. nuestro THA p. 198 ss.

${ }^{45}$ Según nos informa Aristóteles Po. $1457^{\text {a } 35}$ al señalar

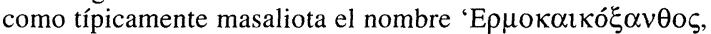
ver los trabajos de Robert, L., «Noms de de personnes et civilisation grecque. I. Noms de personnes dans Marseille grecque», Opera minora selecta, Amsterdam, 1990, VII, p.153 y ss., Masson, O., «Le curieux nom d'un marseillais chez Aristote: Hermokaikoxanthos», Journal des savants 1985, pp. 17-23 y de Hoz, J., «Ensayo ...», cit. (n. 11), 170,

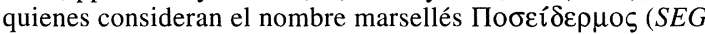
34.1065 , III a.C.) en relación con el río Hermo. Este rasgo es compartido también (ver supra y n. 12) con las colonias eolias minorasiáticas: Escamandro o Escamadrónimo es el pa-

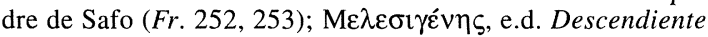
del río Meles es el primer nombre de Homero, a quien se hace nacido en Esmirna y viajero por Etruria e Iberia en la Vida del Ps.-Heródoto, obra ya mencionada aquí; Hecateo (Fr. 227) en su descripción de la franja eólide de Asia Menor (sus Aio $\lambda \iota \kappa \alpha ́$ ) llama al golfo de Esmirna golfo Meleto, es decir del río Meles; cf. Tischler, J., Kleinasiatische Hydronymie. Wiesbaden, 1977 s.vv.

${ }_{46}$ Que se manifiesta cada vez más estrecha a finales del VI: Ugolini, D. y Olive, C., «Grecs et ibères entre l'Orb et l'Hérault (VI-IV a. Ch.) ...», Iberos y griegos..., Eds. Cabrera, P., Olmos, R. y Sanmartí, E., HArq., cit. (n. 14), (2) 295.

${ }_{47}$ Lejeune, M. y Poilloux, J., «Une transaction commerciale ionienne au Ve siècle a Pech-Maho», CRAI, 1988, 526-536, especialmente p. 530. Sobre el nombre de Marsella en la cara A etrusca, ver de $\mathrm{Hoz}, \mathrm{J}$., «L'écriture ibérique», Les ibères, cit. (n. 15), 264; id., «Los negocios del Sr. Heronoiyos», cit.

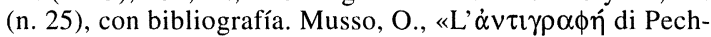
Maho (Aude, Francia) e un'etimologia di Tuscus», Rassegna di studi del civico museo archeologico e del civico gabinetto numismatico di Milano, 51/52, 1993, 39-40 cree que ambas caras son copia la una de otra autorizadas por la misma persona, con deducciones «etimológicas» un tanto arriesgadas.
Pero también en otros antiguos epígrafes griegos encontrados en el ámbito ibérico pueden detectarse huellas de un fluido tráfico con Etruria. En IGAI 4 (EGH 2.56) ${ }^{48}$ contemplamos la intensidad del traspaso de conocimientos técnicos en el Mediterráneo oriental, para lo que debemos retrotraernos una vez más a la tradición hesiódica.

En Hesíodo se empieza a atisbar el abandono de una economía de supervivencia en la que destacan oficios especializados a los que se dedican grupos de personas cada vez en mayor número y entre los que existe gran competencia (Op. 25-6). Entre ellos destaca el alfarero, lo que hizo que pareciera verosímil el adscribir a nuestro poeta el curioso poema

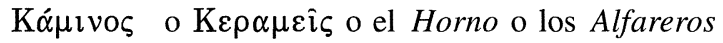
$(F r .302)^{49}$. En esta obra, el poeta está dispuesto a invocar la presencia de Atenea para que propicie buena cochura, correcto ennegrecimiento de los cacharros y buen negocio, si los alfareros le remuneran; si no lo hacen, el aedo conjurará a malévolos genios destructores de los hornos y los objetos que contienen. Este miedo del artesano a que la cochura sea defectuosa, tan hábilmente utilizado por el vate, se traduce en IGAI 4 (EGH 2.56) en búsqueda de soluciones puramente técnicas que impidan la rotura de los vasos durante la cochura o que mantengan el color, especialmente el negro ${ }^{50}$. Una de las soluciones propuestas es probablemente lo que hoy en día llamaríamos espionaje industrial. Énergo, el remitente de la tablilla/carta recomienda a su hermano que estudie los hornos etruscos para conseguir algún dato técnico que impida la temible rotura de

48 Esta inscripción ha suscitado dudas a causa de lo extraño de su aparición y alfabeto, tal como cuenta en la $e d$. $p r$. Dunst, G., «Ein griechisches Tontäfelchen von der Küste bei Ampurias» $\operatorname{MDAI}(M), 10,1969,146-154$; Johnston, A.W., en Suppl. de 1990, p. 465 a Jeffery, L.H., The local Scripts of Archaic Greece. Oxford, 1961, considera que la tablilla puede ser una copia, pero de un texto posiblemente antiguo; se han visto también en ella rastros «des ductus ibériques», ver Dubois, L., Bull.Epigr. 1998, n 569 . A pesar de no ser especialistas en el tema, se nos ocurre, que efectivamente, pueda haber rasgos que evidencien un substrato indígena: ¿posibilidad de que 'E $\mu \pi u ́ \lambda ı$ sov sea una grafía particular sobre una pronunciación nativa de 'E $\mu \pi$ ópıov?

49 El léxico especializado de este poema puede remontar a época micénica, ver Eckstein, F., Archaeologia homerica. Die Denkmäler und das frühgriechische Epos, Gotinga, 1974, 27, aunque no cita para nada a Hesíodo, cf. Morpurgo, A., «Terminology of power and terminology of work in Greek and Linear B», Colloquium Mycenaeum. Actes du $6^{\text {eme }}$ Colloque International sur les textes Mycéniens et Egéens (Chaumont sur Neuchâtel Septembre 1975). Ginebra, 1979. 89 ss. Curiosamente, el poema se encuentra también, atribuido a Homero, en la Vida del Ps.-Heródoto 23, varias veces citada en el presente trabajo.

${ }^{50}$ El editor Dunst, G., «Ein griechisches Tontäfelchen von der Küste bei Ampurias», cit. (n. 49), 146-154 sugiere $\dot{\alpha} \lambda \varepsilon i<\phi>\varepsilon$ iv para la 1.5 . 
las vasijas. Etruria y sus «islas» fueron un importante foco siderúrgico ${ }^{51} \mathrm{y}$ por lo tanto productor de abundante hollín, que debió conformar el «paisaje industrial» que determinó que la isla de Elba fuera llamada por Hecateo en su Fr. 59, Ai $\theta \dot{\alpha} \lambda \eta^{52}$, es de-

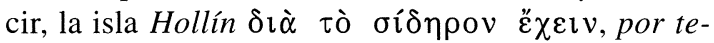
ner hierro. Por lo tanto, tiene cierta lógica que en una época casi contemporánea del historiador, Énergo piense en importar algún hollín especial de Etruria, cuestión que parece haber resuelto localmente, aunque aconseja todavía a su hermano que aproveche el viaje a esa región para investigar los hornos. Énergo no parece ser únicamente un modesto alfarero, sino que testimonia experiencia (¿y actividad?) metalúrgica en relación con el bronce y la plata, con lo que añade un dato local de primera mano a las tradicionales noticias sobre la riqueza minera de la Península, que aparecen también en Hecateo en for-

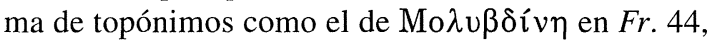

51 «Islas sagradas» según Hesíodo Th. 1016, cf. también Fr. 390; de Elba se llegarán a extraer millones de toneladas de hierro, ver Healy, J.F., Mining and metallurgy in the Greek and Roman world. Londres, 1978, 63.

52 Ver en Diccionario Griego Español cit. (n. 1), s.u., en prensa. del que ya hemos hablado, "I $\beta u \lambda \lambda \alpha$ del Fr. 45 Nen-

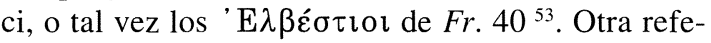
rencia al tráfico con Etruria, aunque dudosa, estaría en el nombre supuestamente etrusco Tı $\mathrm{T} \lambda \alpha \rho\left[{ }^{54} \mathrm{del}\right.$ plomo IGAI 8 B.1 (EGH 2.15).

Debemos poner fin aquí a nuestro caminar al hilo de la Periégesis de Hecateo. Esperemos que nuestro trabajo permita arrojar alguna luz sobre los mecanismos que permitieron al «padre de la Historia», además de ser un hombre $\pi 0 \lambda u \pi \lambda \alpha \nu \eta ́ \varsigma$ gran viajero, conseguir la información necesaria para que su obra fuera considerada «asombrosa» por sus contemporáneos, gracias en parte al activo tráfico testimoniado por las arcaicas y preciadas inscripciones griegas de la Península.

${ }^{3}$ Ver supra y n. 18 y nuestro THA pp. 9 ss., 151; antes que Hecateo, cf. el pasaje de Estesícoro 184 p. 154 Davies.

${ }_{54}$ En la ed. pr., Santiago, R.A. y Sanmartí, E., «Une nouvelle plaquette de plomb trouvée à Emporion», $Z P E, 77$, 1989, [36-38], 38; pero nombre ibérico, Santiago, R. A., «Presencia ibérica en las inscipciones griegas recientemente recuperadas en Ampurias y Pech-Maho», en Iberos y griegos..... Eds. Cabrera, P., Olmos, R. y Sanmartí, E., HArq., cit. (n. 14), (2), 215-230, cf. Faria, A.M., Vipasca, cit. (n. 42), 107.

\section{APÉNDICE DE TEXTOS EPIGRÁFICOS UTILIZADOS}

IGAI 1 ( $E G H 2.14$ ), en una tablilla de plomo. Ampurias, vI$\mathrm{v}$ a.C.
IGAI 2 (EGH 2.38), en la base de una copa. Ampurias, fines del vi a.C.

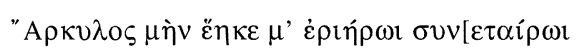

Arcilo me envió a su fiel comlpañero

IGAI 3 (EGH 2.43), en un fragmento cerámico. Ampurias, VI-v a.C.

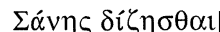

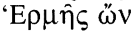

de Sane? buscar ...

siendo Hermes?

IGAI 4 (EGH 2.56), en una tablilla de arcilla. Entre Ampurias y La Escala, 500 a.C.

$$
\begin{aligned}
& \chi \alpha \hat{\imath} \rho \varepsilon,\left(s c . \lambda \varepsilon \dot{\gamma \varepsilon \imath)}{ }^{\prime E v \varepsilon \rho \gamma o \zeta, ~} \dot{\alpha} \delta \varepsilon \lambda \phi o ́ \varsigma\right.
\end{aligned}
$$

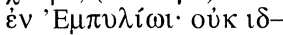

$$
\begin{aligned}
& \text { íov } \pi \eta \lambda \text { oû } \delta \varepsilon \hat{\imath} \varepsilon i ̄ \tau \varepsilon \alpha<\sigma>\beta- \\
& \text { ó } \lambda \mathrm{ov} \cdot \dot{\alpha} \lambda \lambda \dot{\alpha} \gamma \grave{\alpha} \rho \chi \rho \eta<\sigma>\tau \grave{\alpha} \\
& \kappa \alpha i \imath \hat{\omega} \mathrm{l} \chi \alpha \lambda \kappa \hat{\omega} \mathrm{l} \dot{\alpha} \rho \gamma- \\
& \text { ро А } \Pi \text { - са. 5- }
\end{aligned}
$$

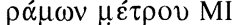

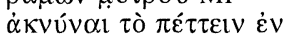

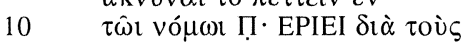$$
5 \quad \kappa \varepsilon ́ \kappa \alpha v \kappa \alpha \dot{\alpha} \lambda \varepsilon \dot{\varepsilon}<\phi>\varepsilon i v,<\ddot{\omega}>\sigma \tau \varepsilon \mathrm{A}
$$ 


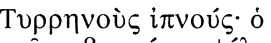

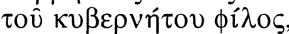

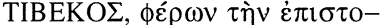

$\lambda \grave{\eta} \nu$ EPEח

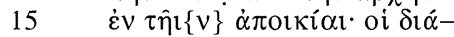

форо $\gamma \lambda \alpha \cup \kappa o i ̀ ~ \kappa \rho \alpha \tau \varepsilon-$

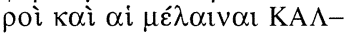

Un saludo (envía) Énergos, (tu) hermano (que vive) en Empilio (Emporion?). No hay necesidad de ningún barro especial ni hollín, pues ya he cocido (vasijas) que son buenas para pintar, del mismo modo que (se trabaja) con bronce en plata (o del mismo modo que se añade la plata al bronce)..... Es decir, que el cocer del modo habitual (con tal porcentaje) hace que se rompan las vasijas. Examina los hornos de tipo etrusco. Tíbeco, el amigo del capitán, que llevará la carta, tiene autoridad (?) en la colonia. Los de calidad superior, verdes y resistentes, $y$ las negras ...

IGAI 5 (EGH 2.16), en una laminilla de plomo. Ampurias, 450- a.C.

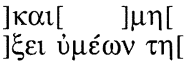

$\kappa \alpha] \grave{i} \Pi v \theta \alpha \gamma o ́ \rho[\eta] \varsigma \dot{\varepsilon}[$

]'A $\gamma \alpha \theta \circ \kappa \lambda \hat{\eta}[\varsigma] \mu \varepsilon[$

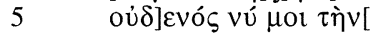

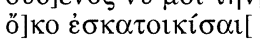

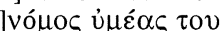

]$\mu \varepsilon \theta \alpha \kappa \alpha \grave{\imath} \tau \hat{\eta} \varsigma \gamma \rho[\alpha \phi \hat{\eta} \varsigma$

... y ... / ... de vosotros ... / ... y Pitágoras ... / ... Agatocles ... / ... de nadie a mí la ... / ... donde establecer ... / ... la ley a vosotros ... / ... y del escrito

IGAI 6 (EGH 2.17), en una lámina de plomo. Ampurias, 450a.C.

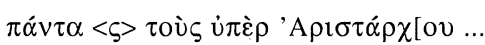

'Apí $\sigma \tau<\alpha \rho>\chi 0 \varsigma$

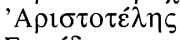

$\sum \omega \sigma i \delta \eta \mu \mathrm{o} \varsigma$

$5 \quad$ 'Елікороৎ

$\Pi \alpha \rho \mu \varepsilon \dot{v} \omega v$

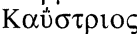

$\Delta \eta \mu \eta \dot{\tau} \tau 10[\varsigma]$

$\Pi v \delta[] \vee \eta \varsigma$

A todos los que están a favor de Aristarco: / Aristarco / Aristóteles / Sosidemo / Epicuro / Parmenón / Caustrio / Demetrio / Pid...es

IGAI 7, en una lámina de plomo. Pech Maho (próximo a Narbona, sur de Francia) ), vi-v a.C.

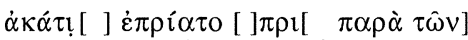

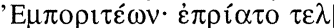
]

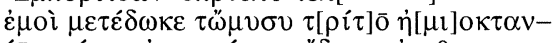

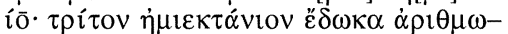

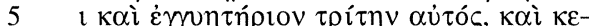
îv'

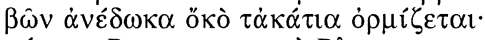

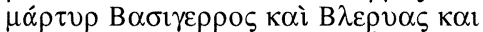

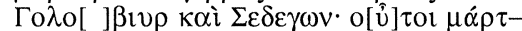

10 vacat $v \rho \varepsilon \varsigma$ $\varepsilon \dot{u} \tau \varepsilon$ خòv $\dot{\alpha} \rho \rho \alpha \beta \hat{\omega} v^{\prime} \dot{\alpha} v \varepsilon ́ \delta \omega \kappa \alpha$,

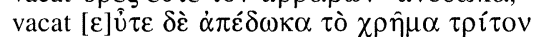

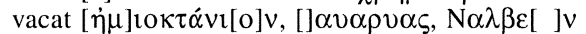

A

'H
... había comprado una barca (¿o barcas?) a los emporitanos, y compró también .... A mí me cedió una participación de la mitad al precio de dos octanios y medio. Dos hectanios y medio le dí en efectivo y una fianza personal de una trite, y aquella suma final la recibió en el río. El anticipo se lo entregué donde están atracadas las barcas, siendo testigos Basigerro, Blerias, Golo.biur y Sedegon; éstos, testigos cuando entregué la señal; pero cuando completé la suma de dos octanios y medio ..auaruas, Nalbe..n Heronoiios?

IGAI 8 (EGH 2.15), en una lámina de plomo. Ampurias, 400 a.C.

A

]$\lambda \imath[$

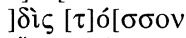

] $\omega \nu[\eta \sigma] \theta[\varepsilon$

]ov óvî $\sigma \alpha 1$

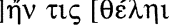

] $\alpha \dot{\tau} \tau \hat{\omega} \mathrm{i} \delta$ [̇े

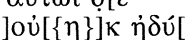

$\operatorname{vos} \dot{\varepsilon} \varsigma^{\prime} \mathrm{A}[\ldots \dot{\eta} \lambda \lambda \theta \varepsilon \ldots]$

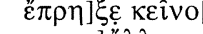
]$\alpha \hat{\alpha} \lambda \lambda$ or $\kappa \alpha i ̀$ 'O[

B

$\pi \alpha \rho] \grave{\alpha} \mathrm{T} \imath \varepsilon \lambda \alpha \rho[$

]$\alpha \sigma \alpha \sigma \lambda[$

]$\alpha \ddot{v} v \delta \rho \alpha v \alpha[\hat{v} \kappa \lambda \eta \rho \circ v$

? I dos veces tanto ... habéis ganado ... / ser ventajoso para .../ 5 ... si alguno (quiere?) ... / ... pero a él ... / ... no agradable ... / ... (?) fue a A... /... llevó aquello a cabo ... / 10 ... O... y otros ... ... para Tielar.. / ...?... / 3 ... armador de barco ...

IGAI 9, en un fragmento de cerámica. Málaga, vi a.C.

['A] $\theta \bar{\varepsilon} v \alpha i \alpha$

Atenea

IGAI 10, en un fragmento de cerámica. Huelva, ca. 550 a.C.

$$
\begin{aligned}
& \text { ] } v \operatorname{vin} \theta \omega \mathrm{i}[ \\
& \text { a Nieto (o Niato?) }
\end{aligned}
$$

IGAI 11, en un fragmento de cerámica. Málaga, vi a.C.

\section{$\Gamma \lambda \alpha \cup \kappa[$}

Glauclo

$I G A I 12$ ( $E G H 16.1$ ), en un fragmento de cerámica ática. Toscanos, Málaga, viı a.C.

$$
\begin{aligned}
& \ldots] \tau o \rho[o \varsigma \\
& \text { de }[\ldots] \text { tor }
\end{aligned}
$$

IGAI 13 (EGH 22.2), en una copa jonia. Huelva, 600-550 a.C.

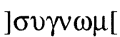

de acuerdo? 
IGAI 14 (EGH 17.1), en dos fragmentos de una copa samia. Guadalhorce, Málaga, 630-600 a.C
a) $] \alpha \tau[$
b) ]ọ $\delta \omega \sigma$
a)...?.../b) dará ?

IGAI 16 (EGH 11.3), en un fragmento de cerámica ática. Guardamar de Segura, Alicante, principios del v a.C.

$$
\Lambda \varepsilon ́ \omega \text { vel ] } \lambda \varepsilon \omega
$$$$
\text { Leo o [...]leo }
$$

IGAI 17 ( $E G H$ 10.1), en un fragmento de cerámica ática. Elche, Alicante, v a.C.

$$
\begin{aligned}
& \kappa о \rho[. . .] \varsigma \kappa \alpha \lambda o ́ \varsigma \\
& \text { guapo muchacho? }
\end{aligned}
$$

IGAI 21 ( $E G H$ 7.1), en una figurilla masculina. Museo de Prehistoria de Valencia, 500 a.C.

'A $\pi \mathrm{o} \lambda<\lambda>\bar{o} \mathrm{VLo \zeta}^{\prime} \alpha \nu \varepsilon \dot{\theta} \theta \bar{\varepsilon} \kappa \varepsilon \nu$

Apolonio (la) ofrendó
IGAI 24 (EGH 2.41), en un fragmento de cerámica. Ampurias, VI-v a.C.

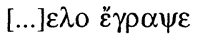

$$
\begin{aligned}
& \text { ]elo lo dibujó }
\end{aligned}
$$

EGH 2.1, en lápida de piedra caliza. Ampurias, I a.C

$$
\begin{aligned}
& \Theta \varepsilon ́ \sigma \pi \mathrm{r}
\end{aligned}
$$

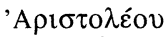

$$
\begin{aligned}
& M \alpha \sigma \sigma \alpha \lambda \imath \eta \dot{\tau} \alpha \\
& \chi \alpha i ̂ p \varepsilon
\end{aligned}
$$

Tespis, hijo de Aristóleo, masaliota, salve.

EGH 2.18, en lámina de plomo. Ampurias, Iv/III a.C.

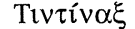

$$
\begin{aligned}
& \Pi о \sigma เ \delta \omega \vee \alpha \varsigma \\
& \text { "О } \rho \tau \omega \nu \\
& 4 \quad \Sigma \omega \sigma i \pi \alpha \tau \rho \circ \varsigma \\
& \text { 'Ерно́кацкоธ } \\
& \Pi \alpha \rho \mu \varepsilon \dot{\varepsilon} \omega v
\end{aligned}
$$

Tintinax, Posidonas, Ortón, Sosipatro, Hermócaico, Parmenón. 